\title{
What's in a name?
}

\section{Why these proteins are intrinsically disordered}

\begin{abstract}
A. Keith Dunker, ${ }^{1}$ M. Madan Babu, ${ }^{2}$ Elisar Barbar, ${ }^{3}$ Martin Blackledge ${ }^{4}$ Sarah E. Bondos, ${ }^{5}$ Zsuzsanna Dosztányi, ${ }^{6}$ H. Jane Dyson, ${ }^{7}$ Julie Forman-Kay, ${ }^{8}$ Monika Fuxreiter, ${ }^{9}$ Jörg Gsponer, ${ }^{10}$ Kyou-Hoon Han, ${ }^{11}$ David T. Jones, ${ }^{12}$ Sonia Longhi, ${ }^{13}$ Steven J. Metallo, ${ }^{14}$ Ken Nishikawa, ${ }^{15}$ Ruth Nussinov ${ }_{1}^{16,17}$ Zoran Obradovic, ${ }^{18}$ Rohit V. Pappu, ${ }^{19}$ Burkhard Rost, ${ }^{20}$ Philipp Selenko, ${ }^{21}$ Vinod Subramaniam, ${ }^{22}$ Joel L. Sussman, ${ }^{23}$ Peter Tompa ${ }^{24-26}$ and Vladimir N. Uversky ${ }^{27,28, *}$
\end{abstract}

\begin{abstract}
'Indiana University School of Medicine; Indianapolis, IN USA; ${ }^{2}$ MRC Laboratory of Molecular Biology; Cambridge, UK; ${ }^{3}$ Oregon State University; Corvallis, OR USA; ${ }^{4}$ Institut de Biologie Structurale CNRS-CEA-UJF; Grenoble, France; ${ }^{5}$ Texas A\&M Health Science Center; College Station, TX USA; ${ }^{6}$ Institute of Enzymology, Hungarian Academy of Sciences; Budapest, Hungary; ${ }^{7}$ The Scripps Research Institute; La Jolla, CA USA; ${ }^{8} \mathrm{Hospital}$ for Sick Children and University of Toronto; Toronto, ON Canada; ${ }^{9}$ University of Debrecen; Debrecen, Hungary; ${ }^{10}$ University of British Columbia; Vancouver, BC Canada; ${ }^{11}$ Korean Research Institute of Bioscience and Biotechnology; Daejeon, Korea; ${ }^{12}$ University College London; London, UK; ${ }^{13}$ Architecture et Fonction des Macromolécules Biologiques; CNRS and Aix-Marseille Université; Marseille, France; ${ }^{14}$ Georgetown University; Washington, DC USA; ${ }^{15}$ Osaka University; Suita, JP; ${ }^{16}$ National Cancer Institute; Frederick, MD USA; ${ }^{17}$ Tel Aviv University; Tel Aviv, Israel; ${ }^{18}$ Temple University; Philadelphia, PA USA; ${ }^{19}$ Washington University in St. Louis; St. Louis, MO USA; ${ }^{20}$ Technischen Universität München; München, Germany; ${ }^{21}$ Leibniz Institute of Molecular Pharmacology; Berlin, Germany; ${ }^{22}$ University of Twente; Enschede, Netherlands; ${ }^{23}$ The Weizmann Institute of Science; Rehovot, Israel; ${ }^{24} \mathrm{VIB}$ Department of Structural Biology; Brussels, Belgium; ${ }^{25}$ Vrije Universiteit Brussel; Brussels, Belgium; ${ }^{26}$ Institute of Enzymology; Budapest, Hungary; ${ }^{27}$ University of South Florida, College of Medicine; Tampa, FL USA; ${ }^{28}$ Institute for Biological Instrumentation; Russian Academy of Sciences; Pushchino, Russia
\end{abstract}

"What's in a name? That which we call a rose

By any other name would smell as sweet."

From "Romeo and Juliet"

William Shakespeare (1594)

This article opens a series of publications on disambiguation of the basic terms used in the field of intrinsically disordered proteins. We start from the beginning, namely from the explanation of what the expression "intrinsically disordered protein" actually means and why this particular term has been chosen as the common denominator for this class of proteins characterized by broad structural, dynamic and functional characteristics.

The common view is that functional proteins or protein domains have unique and stable 3D structures characterized by the relatively fixed positions of their atoms and backbone Ramachandran angles that both vary slightly around their equilibrium positions due to low-amplitude thermal fluctuations. In addition there is another class of functional proteins and protein regions that contain smaller or larger highly dynamic fragments, and some proteins are even characterized by a complete or almost complete lack of ordered structure under physiological conditions (at least in vitro) which appears to be a critical aspect of these proteins' function in vivo. ${ }^{1-7}$ These proteins and protein regions have no single, well-defined equilibrium structure and exist as heterogeneous ensembles of conformers such that no single set of coordinates or backbone Ramachandran angles is sufficient to describe their

*Correspondence to: Vladimir N. Uversky; Email: vuversky@health.usf.edu Submitted: 02/26/13; Accepted: 02/26/13

http://dx.doi.org/10.4161/idp.24157

Citation: Dunker AK, Babu MM, Barbar E, Blackledge M, Bondos SE, Dosztanyi Z, et al. What's in a name? Why these proteins are intrinsically disordered. Intrinsically Disordered Proteins 2013; 1: e24157. conformational properties. These proteins or protein regions that display marked conformational heterogeneity constitute a significant part of the protein kingdom and they have, over the years, been described in the literature by a plethora of different names, which had been proposed before it was established that this class of proteins constitutes a separate and important extension to the protein kingdom. Indeed, these highly dynamic proteins with important biological functions were independently discovered multiple times, with the authors frequently inventing new terms to describe their protein of interest. As a result, the complex and lengthy route to recognizing these proteins as a novel class left in its path a trail of terms used for their description. Figure 1 depicts a collection of names that can be found in the literature describing these proteins, which includes terms such as floppy, pliable, rheomorphic, ${ }^{8}$ flexible, ${ }^{9}$ mobile, ${ }^{10}$ partially folded, ${ }^{11}$ natively denatured, ${ }^{12}$ natively unfolded, ${ }^{3,13}$ natively disordered, ${ }^{6}$ intrinsically unstructured, ${ }^{2,5}$ intrinsically denatured, ${ }^{12}$ intrinsically unfolded, ${ }^{13}$ intrinsically disordered, ${ }^{4}$ vulnerable,${ }^{14}$ chameleon, ${ }^{15}$ malleable ${ }^{16} 4 \mathrm{D},{ }^{17}$ protein clouds, ${ }^{18}$ dancing proteins, ${ }^{19}$ proteins waiting for partners, ${ }^{20}$ and several other names often representing different combinations of "natively/naturally/inherently/intrinsically" with "unfolded/unstructured/disordered/denatured" among several others. Therefore, the majority of the names used in the early literature express that the "unfolded, unstructured, disordered, and denatured" state is a "native, natural, inherent, and intrinsic" property of these proteins. Strikingly, the idea of protein clouds had an unusual development when protein stucture networks (or protein contact networks) were recently classified into "cumulus-type" (similar to puffy, white clouds) and "stratus-type" (similar to flat, dense, dark, low-lying clouds), depending on protein disorder dynamics and the mechanisms of conformational changes. ${ }^{21}$

The multitude of expressions used to describe this class of proteins in earlier publications reflects the difficulties faced by researchers trying to find an appropriate way to describe these "abnormal" proteins. It also reflects an important recognition 


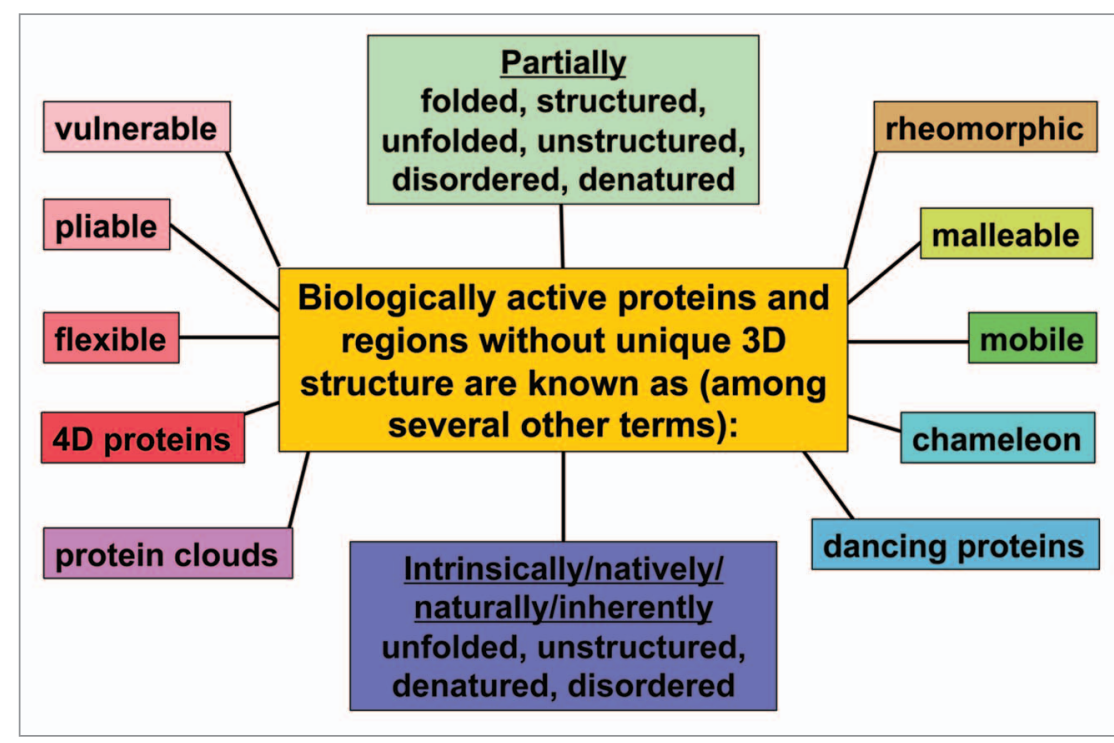

Figure 1. Protein intrinsic disorder Tower of Babel: some of the terms used in the literature to describe biologically active proteins without unique structures. shared vocabulary and taxonomy helps to break the "Tower of Babel" miscommunication, where specialists working on similar subjects cannot understand each other due to the use of different "languages"; i.e., utilizing different terms and definitions invented to describe the same phenomenon. Below we provide some arguments supporting the use of the term "intrinsically disordered proteins" (IDPs) as a general descriptor of biologically active proteins lacking unique 3D-structures.

Many IDP-related terms have very specific meanings or express a unique characteristics but are not general enough (e.g., rheomorphic, ${ }^{8}$ vulnerable, ${ }^{14}$ proteins waiting for partners, ${ }^{20}$ etc.), while some of the other terms are more poetic than scientific (e.g., protein-chameleon, ${ }^{15}$ protein clouds, ${ }^{18}$ dancing proteins, ${ }^{19}$ etc.). Although these terms can be used to emphasize some of the characteristics of such proteins or to attract the attention of a wider audience to protein intrinsic disorder, they are that these proteins differ from globular proteins and likely belong to a separate class. Although some of the terms were romantic (e.g., "vulnerable," "chameleon," "protein cloud" or "dancing protein"), all of them were inspired by the recognition of the existence of a fundamental difference between biologically active, but non-rigid proteins and "classic" globular, transmembrane, and fibrous proteins that can be described by the classical structure-function paradigm, where a unique sequence defines a unique 3D-structure crucial for that protein function.

These "abnormal" proteins did not yield unique 3D-structures. Furthermore, conformational properties of these proteins or protein regions resembled partially folded or completely unfolded conformations found for ordered proteins during the folding process or under various denaturing conditions. Hence, terms to designate these "new" proteins often contained corresponding structure-based descriptors, such as "denatured," "unfolded," "unstructured," "partially folded” or "partially denatured." It was also clear that the aforementioned fundamental difference is native, natural or intrinsic to these proteins representing an inherent property of their amino acid sequences. Therefore, many early terms contained related descriptors, such as "natively," "naturally" or "intrinsically." Besides structural considerations, the different backgrounds of the various researchers also contributed to the diversity of the proposed terms. In fact, since IDPs are of interest to scientists with very different backgrounds, and since each scientific discipline has its own language, with special terms and jargon, new descriptors rooted in these corresponding backgrounds have also been proposed, further complicating the picture by generating too many terms describing similar (or even identical) phenomena.

We, as Editors of the Intrinsically Disordered Proteins journal, feel that the development of a common ontology, that is a formal and explicit description of shared concepts, is an important step needed for the future development of the field, since such a rather specific and do not serve the purpose of communicating the general idea behind the intrinsic disorder phenomenon or identifying these specific proteins as belonging to a related class of proteins. A good general term should contain an adjective to emphasize the uniqueness and specificity of the phenomenon and an informative descriptor (or qualifier) that clearly defines the phenomenon. From the beginning, the choice of a general adjective was limited to three terms, "natively," "intrinsically" and "naturally." The adjective 'natively' has obvious functional meanings, as it reflects the important fact that IDPs lack unique structure in their native (i.e., biologically active) states. On the other hand, the lack of classical 3D structures represents an "intrinsic" or "natural" property of a protein, because it is encoded in its primary amino acid sequence and further defined by its sequence-specific, compositional bias. Since these three adjectives ("natively," "intrinsically" and "naturally") describe the inherent nature and uniqueness of the phenomenon equally well, there is no obvious advantage in using one expression over the other and so all three adjectives are found throughout the (early) literature. With time, however, preference was given to the term 'intrinsic', because it nicely conveys the notion that the behavior of the polypeptide chain is primarily defined by its amino acid sequence, which under generic physiological conditions shows a preference for a distinct, albeit broad distribution of non-folded conformational states. The details of this distribution can be very different for various disordered proteins. Also, it is difficult to know a priori whether in vitro observed characteristics of IDPs are similarly displayed in cellular environments. Therefore, 'intrinsic' refers to the innate tendencies of these proteins in isolation and under physiological conditions.

Choosing an informative structural qualifier is more difficult and a collections of terms have been used in the literature: "flexible," "dynamic," "heterogeneous," "denatured," "unstructured," "unfolded" and "disordered." To choose the most appropriate 
descriptor one must consider the global meaning of the terms. From this viewpoint, we would argue that "dynamic" and "flexible" are probably not the expressions that we seek. In fact, the use of the word dynamics or "dynamic" as a preface for a descriptor presumes prior knowledge of the time scales on which conformational conversions occur. It is also worth noting that even techniques that probe conformational conversions on distinct time scales are actually reporting on an ensemble average of signals. On the other hand, disordered proteins studied by NMR display NMR characteristics that are consistent with dynamic interconversion between conformations on timescales ranging from picoseconds to milliseconds, depending on the line-shapes. Given this observation, one would venture that a statement such as "IDPs are dynamic" could be considered to be a given. However, since all proteins (intrinsically disordered and ordered) are dynamic to different degrees in terms of amplitudes and timescales, the descriptor 'dynamic' is not a useful term for IDPs.

Similarly, all proteins are intrinsically flexible to some extent (i.e., they have varying degrees of conformational freedom) and this intrinsic flexibility is often vital for their functions. Enzymes, for example, typically contain "flexible," but well-structured pockets or arms that are essential for binding to substrates, or ligands. On the other hand, with only a few noticeable exceptions ${ }^{22}$ these proteins are not intrinsically disordered and rather possess well-defined, three-dimensional structures with flexible regions or appendages. Traditionally, the term "flexibility" is used to describe the magnitude of fluctuations around some equilibrium geometry. IDPs are structurally heterogeneous and do not have such "equilibrium geometry." Therefore, strictly speaking, the term "flexibility" is not applicable for IDPs, whose structures are described as conformational ensembles. In fact, each member of these conformational ensembles is characterized by its own "flexibility."

It is also worth noting that the intrinsic flexibility of a polymer is primarily governed by the nature of the bonds that connect its repeating units. All proteins use the same types of amino acids as building blocks and all amino acids in all proteins are connected in the same manner; i.e., via peptide bonds. However, compared with globular proteins disordered proteins have distinct amino acid compositions that encode a much wider range of conformational and structural heterogeneity. It is the amplitudes and timescales that are associated with spontaneous conformational fluctuations under similar solvent conditions that set apart one group of protein sequences from another. Hence, it is the collective nature of the interactions rather than the intrinsic flexibility that matters. And the impact of this collectivity of interactions is on the nature of conformational distributions, which becomes the important discriminator among different IDPs.

The terms "denaturation" and "denatured" are derived from the behavior of ordered macromolecules (including proteins) under conditions that lead to a loss of function due to disruptions of their tertiary and/or secondary structures. Denaturation can be induced by a variety of environmental factors or external stresses such as strong acids or bases, increased or decreased temperatures, concentrated inorganic salts, strong denaturants (e.g., urea or guanidinium), and some organic solutes (e.g., alcohols, etc.). The term "natively denatured" is a very clever juxtaposition and was introduced to emphasize the existence of drastic structural differences between 'normal' globular proteins, with rigid tertiary structures, and "abnormal" flexible proteins such as tau. ${ }^{12}$ However, since by definition, "denatured" means "nonfunctional" and implies the existence of a "non-denatured, native, functional" state, which does not exist in the case of IDPs, this term does not suit the purpose of describing proteins that do not have unique structures but do have specific biological functions. Furthermore, denaturation of ordered proteins in a living cell usually results in disruption of cell activity and possibly cell death, while many IDPs are crucial for cell survival.

The terms "unstructured" and "unfolded" were used extensively in early publications dedicated to IDPs. This was mostly due to the fact that the majority of IDPs analyzed at that time happened to be unstructured; i.e., they did not have unique 3D-structure and contained very little ordered secondary structure. The discovery and characterization of such "structure-less" proteins was important as a counter-point to the hitherto dominant view represented by the classical structure-function paradigm, especially since such fully unstructured, yet functional, proteins clearly represented the other extreme of the protein structure spectrum. These two terms are synonymously used and frequently considered to relate to the expression 'random coil' that is used in polymer physics to describe the conformational state of a polymer under conditions where the monomer subunits are well solvated and oriented randomly while still being bonded to adjacent units. However, it is important to keep in mind that due to their heteropolymeric nature, proteins are never in completely random conformations and always have some residual structure. In fact, there is no such thing as absence of structure in an aqueous solution of a polypeptide. There are always some preferential conformations sampled, and certainly a preference for an extended structure does not imply that the chain is "random" or "structure-less." That proteins are never true "random coils" applies to both ordered proteins under highly denaturing conditions and to IDPs under physiological conditions. ${ }^{23-29}$ Of course, one can argue that the term "random coil" can still be used to describe an IDP whose structural features are close to those of unfolded ordered proteins under strong denaturing conditions. The counterargument here is the extreme structural heterogeneity of IDPs: although this term is suitable for the description of so-called extended IDPs (i.e., native coils and native pre-molten globules, which were and are commonly defined in the literature as natively unfolded or intrinsically unstructured protein $s^{5,30}$ ), it definitely is a poor qualifier for compact or collapsed IDPs (i.e., native molten globules).

This leaves us with the expression 'disordered', which is often used to compare unique, well-defined states of proteins and situations in which proteins adopt many different structural ensembles, with no single, preferred lowest energy conformation. ${ }^{31}$ Therefore, the term "disordered" also entails the ensemble description of structurally heterogeneous populations, with different levels of internal heterogeneity. It is important to understand that different degrees of structural heterogeneity are inherently present in all protein populations, but that its 
preponderance is significantly higher in IDPs. From this viewpoint, biologically active proteins without unique $3 \mathrm{D}$-structures can be considered as intrinsically heterogeneous. Another issue is that some degree of structural heterogeneity is also inherent to a well-folded protein. In fact, "heterogeneity" is a term applicable over a range of heterogeneous states, whereas "disorder" is much more applicable to highly heterogeneous states. Therefore, for an accurate designation of the nature of heterogeneity/degree-ofdisorder, one needs at least two order parameters, the density or $\mathrm{R}_{\mathrm{g}}$ (gyration radius or any other measure of protein compaction) and a measure of the amplitudes of fluctuations. And the more we dig, the more we find that no single phrase will capture the full spectrum of sequence-to-ensemble relationships.

Obviously, due to the depth and breadth of this newly recognized phenomenon, where "structurelessness" might show itself differently in different proteins and might affect different levels of protein structural organizations to different degrees, none of the terms proposed for defining these biologically active proteins is perfect. Also, although the ideal term should contain a precise description of the phenomenon, the chances of finding/inventing such a term are very slim if the phenomenon is very broad. In fact, already in some of the first reviews on this topic it was pointed out that it might be difficult to describe this new class of proteins with a single term. ${ }^{4,30}$ Despite all these difficulties, the term "intrinsically disordered proteins" (IDPs) is currently used more often than any other expression, probably because of the simple logic that nothing is perfect and that "IDP" represents an acceptable compromise. We believe that the use of a single common term to describe these proteins is the best option. Common use of a single descriptor will eventually pay off, even if a chosen term is somewhat far from being ideal. Time will smooth the ambiguities and roughness of the original term and its true meaning will become clear. This will unify the field and we trust that, with time, inconsistencies will be smoothed out by additional descriptors, such as "extended IDPs," for example.

To conclude this discussion, we also propose that based on the structural classifications of IDPs, the annotation "disordered protein" may be applied to any set of non-rigid independent conformations of a polypeptide chain and the term "protein intrinsic disorder" could be used to describe the phenomenon. We also suggest that the expression "unstructured proteins," if used, should only be applied to the subset of disordered proteins, socalled extended disordered proteins (e.g., native coils or native pre-molten globules), which are characterized by the absence of any (or almost any) ordered structure (although one clearly should keep in mind that there is always some preferential structure even in the most extended IDPs). ${ }^{30}$

Obviously, a protein is called ordered when it does not contain any intrinsic disorder, and it is called intrinsically disordered if the entire protein does not contain any tertiary structure. A majority of eukaryotic protein sequences are chimeras of ordered and disordered regions and the synergy between these regions determines the range of protein functions in different cellular contexts. Therefore, a very important question is how one can name a mixed or hybrid protein that has both ordered and disordered regions. We believe that calling such a protein 'disordered' is misleading, similiar to referring to it as "ordered." In fact, such a protein does not fall into either category and therefore should be classified as a hybrid protein or as a protein that has intrinsically disordered regions (IDRs) or intrinsically disordered domains. Here, IDRs are disordered regions within protein sequences whose amino acid compositions prevent them from autonomously folding into defined 3D structures. This of course immediately raises the ensuing question as to how long a disordered region must be to qualify as such. Clearly two or three disordered residues, typically at protein termini, do not merit to be considered as a disordered region. A length of 20-30 residues seems to be a reasonable limit, at least in terms of justifying efforts aimed at characterizing the conformational and dynamic properties of such a region. Finally, one should keep in mind that intrinsic disorder takes many forms $s^{4,732-34}$ and that a continuum of differently disordered conformations/states exists in the protein realm (e.g., see Fig. 1 in ref. 32 and Fig. 4 in ref. 34).

As a last note, we would like to emphasize that it is difficult to overestimate the importance of clarity in how the scientific community describes IDPs/IDRs, since these proteins/regions, being crucial for various biological processes, are abundant in all the proteomes and are commonly associated with the development of various pathologies.

\section{References}

1. Dunker AK, Garner E, Guilliot S, Romero P, Albrecht $\mathrm{K}$, Hart J, et al. Protein disorder and the evolution of molecular recognition: theory, predictions and observations. Pac Symp Biocomput 1998; 473-84; PMID:9697205.

2. Wright PE, Dyson HJ. Intrinsically unstructured proteins: re-assessing the protein structure-function paradigm. J Mol Biol 1999; 293:321-31; PMID:10550212; http://dx.doi.org/10.1006/jmbi.1999.3110.

3. Uversky VN, Gillespie JR, Fink AL. Why are "natively unfolded" proteins unstructured under physiologic conditions? Proteins 2000; 41:415-27; PMID:11025552; http://dx.doi. org/10.1002/1097-0134(20001115)41:3<415::AIDPROT130>3.0.CO;2-7.

4. Dunker AK, Lawson JD, Brown CJ, Williams RM, Romero P, Oh JS, et al. Intrinsically disordered protein. J Mol Graph Model 2001; 19:26-59; PMID:11381529; http://dx.doi.org/10.1016/S1093-3263(00)00138-8.
5. Tompa P. Intrinsically unstructured proteins. Trends Biochem Sci 2002; 27:527-33; PMID:12368089; http://dx.doi.org/10.1016/S0968-0004(02)02169-2.

6. Daughdrill GW, Pielak GJ, Uversky VN, Cortese MS, Dunker AK. Natively disordered proteins. In: Buchner J, Kiefhaber T, eds. Handbook of Protein Folding. Weinheim, Germany: Wiley-VCH, Verlag GmbH \& Co. KGaA, 2005:271-353.

7. Uversky VN, Dunker AK. Understanding protein nonfolding. Biochim Biophys Acta 2010; 1804:1231-64; PMID:20117254; http://dx.doi.org/10.1016/j.bbapap.2010.01.017.

8. Holt C, Sawyer L. Caseins as rheomorphic proteins: interpretation of primary and secondary structures of the as1-, b-, and k-caseins. J Chem Soc, Faraday Trans 1993; 89:2683-92; http://dx.doi.org/10.1039/ $\mathrm{ft} 9938902683$.
9. Pullen RA, Jenkins JA, Tickle IJ, Wood SP, Blundell TL. The relation of polypeptide hormone structure and flexibility to receptor binding: the relevance of X-ray studies on insulins, glucagon and human placental lactogen. Mol Cell Biochem 1975; 8:5-20; PMID:170505; http://dx.doi.org/10.1007/BF01731645.

10. Cary PD, Moss T, Bradbury EM. High-resolution proton-magnetic-resonance studies of chromatin core particles. Eur J Biochem 1978; 89:475-82; PMID:710406; http://dx.doi.org/10.1111/j.1432-1033.1978. tb12551.x.

11. Linderstrom-Lang K, Schellman JA. Protein structure and enzyme activity. In: Boyer PD, Lardy $\mathrm{H}$, Myrback $\mathrm{K}$, eds. The Enzymes. New York: Academic Press, 1959:443-510.

12. Schweers O, Schönbrunn-Hanebeck E, Marx A, Mandelkow E. Structural studies of tau protein and Alzheimer paired helical filaments show no evidence for beta-structure. J Biol Chem 1994; 269:24290-7; PMID:7929085. 
13. Weinreb PH, Zhen W, Poon AW, Conway KA, Lansbury PT Jr. NACP, a protein implicated in Alzheimer's disease and learning, is natively unfolded. Biochemistry 1996; 35:13709-15; PMID:8901511; http://dx.doi.org/10.1021/bi961799n.

14. Chen J, Liang H, Fernández A. Protein structure protection commits gene expression patterns. Genome Biol 2008; 9:R107; PMID:18606003; http://dx.doi. org/10.1186/gb-2008-9-7-r107.

15. Uversky VN. A protein-chameleon: conformational plasticity of alpha-synuclein, a disordered protein involved in neurodegenerative disorders. J Biomol Struct Dyn 2003; 21:211-34; PMID:12956606; http://dx.doi.org/10.1080/07391102.2003.10506918.

16. Fuxreiter M, Tompa P, Simon I, Uversky VN, Hansen JC, Asturias FJ. Malleable machines take shape in eukaryotic transcriptional regulation. Nat Chem Biol 2008; 4:728-37; PMID:19008886; http://dx.doi. org/10.1038/nchembio.127.

17. Tsvetkov P, Asher G, Paz A, Reuven N, Sussman JL Silman I, et al. Operational definition of intrinsically unstructured protein sequences based on susceptibility to the 20S proteasome. Proteins 2008; 70:135766; PMID:17879262; http://dx.doi.org/10.1002/ prot. 21614

18. Dunker AK, Uversky VN. Drugs for 'protein clouds': targeting intrinsically disordered transcription factors. Curr Opin Pharmacol 2010; 10:782 8; PMID:20889377; http://dx.doi.org/10.1016/j. coph.2010.09.005.

19. Livesay DR. Protein dynamics: dancing on an everchanging free energy stage. Curr Opin Pharmaco 2010; 10:706-8; PMID:20951643; http://dx.doi. org/10.1016/j.coph.2010.09.015.

20. Janin J, Sternberg MJE. Protein flexibility, not disorder, is intrinsic to molecular recognition. F1000 Biol Rep 2013; 5:2; PMID:23361309; http://dx.doi org/10.3410/B5-02
21. Csermely P, Sandhu KS, Hazai E, Hoksza Z, Kis HJ, Miozzo F, et al. Disordered proteins and network disorder in network descriptions of protein structure, dynamics and function: hypotheses and a comprehensive review. Curr Protein Pept Sc 2012; 13:19-33; PMID:22044146; http://dx.doi. org/10.2174/138920312799277992.

22. Zambelli B, Cremades N, Neyroz P, Turano P, Uversky $\mathrm{VN}$, Ciurli S. Insights in the (un)structural organization of Bacillus pasteurii UreG, an intrinsically disordered GTPase enzyme. Mol Biosyst 2012 8:220-8; PMID:21922108; http://dx.doi.org/10.1039/ c1 mb05227f.

23. Fuxreiter M, Simon I, Friedrich P, Tompa P. Preformed structural elements feature in partner recognition by intrinsically unstructured proteins. J Mol Biol 2004; 338:1015-26; PMID:15111064; http://dx.doi. org/10.1016/j.jmb.2004.03.017.

24. Dyson HJ, Wright PE. Unfolded proteins and protein folding studied by NMR. Chem Rev 2004; 104:3607 22; PMID:15303830; http://dx.doi.org/10.1021/ cr030403s.

25. Mittag T, Forman-Kay JD. Atomic-level characterization of disordered protein ensembles. Curr Opin Struct Biol 2007; 17:3-14; PMID:17250999; http://dx.doi. org/10.1016/j.sbi.2007.01.009.

26. Lee SH, Kim DH, Han JJ, Cha EJ, Lim JE, Cho YJ, et al. Understanding pre-structured motifs (PreSMos) in intrinsically unfolded proteins. Curr Protein Pep Sci 2012; 13:34-54; PMID:22044148; http://dx.doi. org/10.2174/138920312799277974.

27. Marsh JA, Forman-Kay JD. Ensemble modeling of protein disordered states: Experimental restraint contributions and validation. Proteins 2011; 80:556-72; PMID:22095648.
28. Schneider R, Huang JR, Yao MX, Communie G, Ozenne V, Mollica L, et al. Towards a robust description of intrinsic protein disorder using nuclear magnetic resonance spectroscopy. Mol Biosyst 2012; 8:5868; PMID:21874206; http://dx.doi.org/10.1039/ c1mb05291h.

29. Uversky VN. Intrinsically disordered proteins may escape unwanted interactions via functional misfolding. Biochim Biophys Acta 2011; 1814:693-712; PMID:21440685; http://dx.doi.org/10.1016/j.bbapap.2011.03.010.

30. Uversky VN. Natively unfolded proteins: a point where biology waits for physics. Protein Sci 2002; 11:739 56; PMID:11910019; http://dx.doi.org/10.1110/ ps.4210102.

31. Baranger M. Chaos, complexity, and entropy - A physics talk for non-physicists. 2001.

32. Dyson HJ, Wright PE. Intrinsically unstructured proteins and their functions. Nat Rev Mol Cell Biol 2005; 6:197-208; PMID:15738986; http://dx.doi. org/10.1038/nrm1589.

33. Dunker AK, Obradovic Z. The protein trinity--linking function and disorder. Nat Biotechnol 2001; 19:8056; PMID:11533628; http://dx.doi.org/10.1038/ nbt0901-805.

34. Uversky VN. Unusual biophysics of intrinsically disordered proteins. Biochim Biophys Acta 2013; PMID:23269364. 